\title{
HYDROLOGISCH-MORPHOLOGISCHE EINDRÜCKE AUS ANATOLIEN 1
}

\author{
VALENTIN BingGeli
}

Alles ist aus dem Wasser entsprungen!

Alles wird durch das Wasser erhalten! (Goethe)

Wiederholt schon wurde als das eine Leitmal des Orients - neben dem Minarett als dem des Islams - das Dromedar bezeichnet, Sinnbild der steppenweiten Wasserarmut. In der Tat ist das Wasserproblem ein Grundproblem des Orients. Gerade auch in Anatolien werden in den letzten Jahrzehnten Anstrengungen zu Bewässerung, Urbarisierung, Bodenverbesserung des Steppenhochlandes unternommen. Es bestehen zahlreiche Mehrjahrespläne, verzeichnet in der Türkiye Su Hartasi der «Türkischen Waskarte» ${ }^{2}$.

Die besondere Bedeutsamkeit des Wassers erhellt daraus, da $B$ die orientalische Religion der Muselmanen bezeichnenderweise die «Religion des Wassers» genannt wird. Der gläubige Moslem befolgt streng die alte koranische Sitte der Waschung vor jedem der fünf täglichen Gebete 3. Die Bedeutung, die der Orientale dem Lebenselement beimißt, die fast Verehrung zu nennende Haltung dem Wasser gegenüber, äußert sich in einem unerhört kunstvollen Brunnenbau (Fig. 1). Die Brunnen sind häufig moscheenartig mit kleinen Kuppeln versehen, häufig auch ganze kleine Häuser, tragen nicht selten Reliefs und prächtige arabische Schriftsteine. Die bekannte Schönheit altrömischer Aquädukte bieten Istanbul, Kayseri und andere geschichtliche Siedelungen. In Istanbul erregt Erstaunen die antike unterirdische Zisterne (Yerebatan Saray, versunkener Palast), das Notzeit-Wasserreservoir der alten Weltstadt, erbaut im 6. Jahrhundert n. Chr. durch Justinian. 336 Säulen tragen die 8 Meter hohe Gewölbedecke des Raums, mit einer Länge von $140 \mathrm{~m}$ und einer Breite von $70 \mathrm{~m}$, der $70000 \mathrm{~m}^{3}$ Wasser zu fassen vermochte. Über Aquädukte wurde das Wasser $35 \mathrm{~km}$ aus Westen aus dem Belgraderwald hieher geleitet.

Mit dem Mangel an fließendem Oberflächenwasser steht im Zusammenhang eine oft schlechte Wasserqualität. Ins Straßenbild gehört der Wasserverkäufer und der Wasserkrug (Fig. 2). Einigermaßen gutes Trinkwasser bieten nur Städte mit neueren Wasserversorgungsanlagen, die Quellen der Gebirge und Grundwasserreservoire. 6 von Furon, Huegi und Westerveld ${ }^{4}$ studierte, über ganz Anatolien verteilte Gebiete verfügen alle über «gewisse Grundwasservorräte in oberflächennahen Schichten», die intensiver genutzt werden könnten.

\section{GEOLOGISCHE GRUNDZÜGE}

Mannigfaltig sind die geologischen Grundlagen der türkischen Morphologie, von den Küstenlandschaften über die Hochgebirge Taurus und Pontus zu den Steppenhochländern des Innern. Vulkanische Erscheinungen und junge Bruchtektonik (Erdbeben) charakterisieren zusammen mit den randlichen alpinen Faltenzonen ${ }^{5}$ den Bau Kleinasiens.

Am geologischen Aufbau des Steppeninnern, das die Hauptfläche Anatoliens ausmacht, sind vor allem Süßwassersedimente des Tertiärs beteiligt, demnach jüngere Bildungen (Nummulithenkalke, Konglomerate, Sandstein und Mergel, zum Teil also wie bei uns Molassesedimente). Im Miocän und Oligocän kommen Gips und Steinsalz vor. Man nimmt an, die hie und da fensterartig aufgeschlossenen präpaläozoischen Granite, Gneise und kristallinen Schiefer

1 Türkei-Exkursion Herbst 1958 des Geogr. Instituts der Universität Bern unter Leitung von Prof. Dr. F. Gygax.

2 Karte der Wasserwerke und Bewässerungsprojekte der Türkei. 1951.

3 Glasenapp H. v.: Die nichtchristlichen Religionen. Frankfurt a. M. 1957.

4 Hớl TH.: Hydrogeologische Beobachtungen in Anatolien. Ecl. Geol. Helv., Vol. 46, 2, 1953.

- Staub R.: Der alpine Kettenplan Europas, in neuer Gliederung (Karte 1:15000), Ecl. Geol. Helv. Vol. 44, 1951. 
bildeten den Sockel der ganzen Halbinsel. Darüber folgt das ebenfalls unregelmäßig über Anatolien verteilte Paläozoikum, vor allem Silur, Devon und Karbon (Grauwacken, Sandsteine, Kalke), dann folgen die mesozoischen Kalke der Formationen Trias, Jura und der dominierenden Kreide, die vorherrschend am Aufbau der Randgebirge Pontus und Taurus beteiligt sind.

Aus den zahlreichen vulkanischen Gebieten Anatoliens ist hervorzuheben der legendenhafte Ararat, $5165 \mathrm{~m}$, an der Ostgrenze, und der ebenfalls hochgebirgige, erloschene Erciyas Dag, $3916 \mathrm{~m}$, dessen Vulkangebiet eine Fläche von nahezu $10000 \mathrm{~km}^{2}$, demnach einem Viertel derjenigen der Schweiz, bedeckt. Von Chaput 6 wird er als postpontisch angegeben. Er soll als «Mons Argaeus» noch zur Zeit Strabos, in 1. Jahrhundert n. Chr., tätig gewesen sein.

Mit dem Vulkanismus in wechselseitigem Bedingungsverhältnis steht die jungc Bruchtcktonik, dominierende Erscheinung der Geologie Inneranatoliens wie auch besonders des Marmarameers und Aegaeisraums. Am Erciyas Dag beobachtete Prof. GyGAX Verwerfungen in Schliffflächen, also glazial- oder postglazialzeitliche. Die zahlreichen morphologischen Becken des Innern wurden als Einbruchsbecken erkannt, die infolge Absenkung entlang von Kesselbrüchen gebildet wurden. Die große Konya ovasi (Ebene von Konya) zerfällt beispielsweise in 1o Teilbecken, getrennt durch Neogenkalk-Schwellen, wobei jedes dieser Becken eine Stadt als anthropogeographisches Zentrum aufweist. Eine verästelte Bruchanlage zeigt der Marmarameerraum, ebenfalls verläuft konform einer Störungslinie, der wichtigen «nordanatolischen Bebenlinie» (ca. $100 \mathrm{~km}$ nördlich davon), die Schwarzmeer-Küste. Interesse erregt der eigenartige und sicher wie die Küsten bruchtektonisch bedingte Verlauf anatolischer Flüsse. Diese sind oft zusammengesetzt aus mehreren, rechtwinklig stehenden Laufstücken. Als Beispiel demonstriert dies der Sakaria im Westen. Es dürfte sich um ein System rechtwinkliger Brüche und Klüfte handeln, denen die Flüsse folgen, ein ungefähr Ost-West verlaufendes (alpin) und ein solches quer dazu (erythräisch).

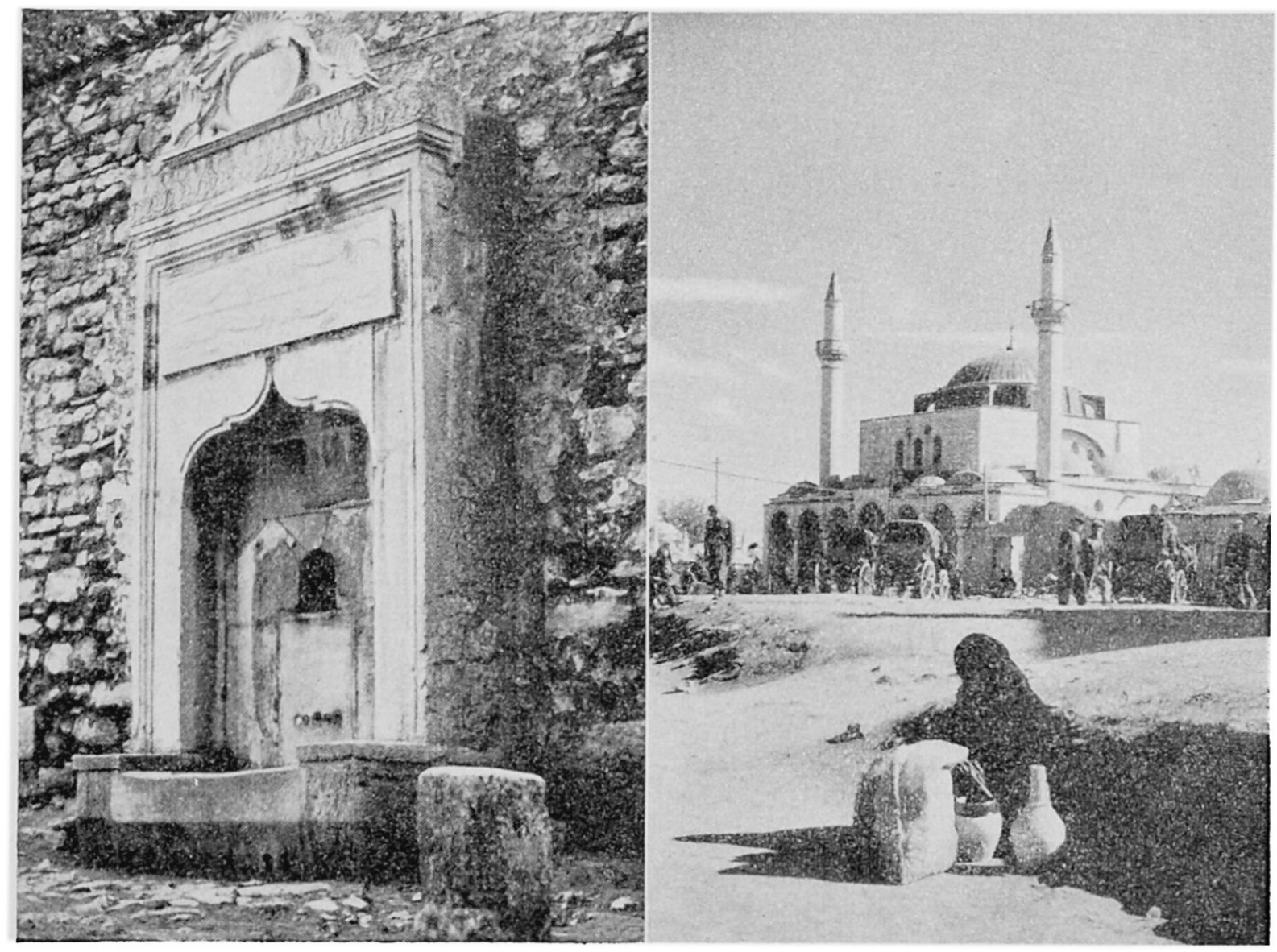

Links Fig. 1: Henkerbrunnen und Scharfrichterstein beim Eingangstor zum Serail, dem alten Sultanspalast in Istanbul. Der prächtige Brunnenbau ist keine Besonderheit, stellt eher eine typische Erscheinung dar, in welcher sich die verehrende Haltung des Orientalen dem Lebenselement Wasser gegenüber äußert. Rechts Fig. 2: Der Wasserkrug, das unbedingte Attribut des Orients. Moschee in Konya. Aufn. Val. Binggeli

6 Chaput E.: Voyages d'Etudes Géologiques et Géomorphologéniques en Turquie. Mem. d. l'Inst. Franç. d'Arch. d. Stamboul. Paris 1936. 
Unter dem Kapitel Erdbeben sei vor allem eben auf die nordanatolische Bebenzone hingewiesen. Hier kann jüngste Bruchbildung studiert werden. So waren die Folgen des ErzincanGroßbebens vom 28.Dezember 1939 nicht nur 40000 menschliche Opfer, es bildeten sich zudem Brüche von Hunderten von Kilometern Länge, mit Sprunghöhen in der Größenordnung von Metern, weiter Bergstürze, Aufstauung von Seen, Austritt von Mineralquellen. Das Erdbeben von Tosya-Ilgaz am 28. November 1943 hatte eine Bruchzone von $280 \mathrm{~km}$ Länge, mit einer relativen Absenkung der Nordseite von ca. $1 \mathrm{~m}$ zur Folge. Die am 1. Februar 1944 entstandene $120 \mathrm{~km}$ lange Bruchlinie wies eine Horizontal-(Blatt-) Verschiebung von 3-5 $\mathrm{m}$ auf. Wiederum vollführte den Abwärts-Sprung die Nordseite, um o,4-1 m (KetIN und RoesLI) 7.

\section{DIE KLIMATISCH - HYDROLOGISCHE HETEROGENITÄT}

Kontrastreiche Vielfalt herrscht auch in klimatischer und hydrologischer Hinsicht. Dem kontinentalen des Innern stehen das mediterrane Meerklima und die Gebirgsklimate von Taurus und Pontus gegenüber. Fig. 3 und 4 zeigen für den Niederschlag ${ }^{8}$ die größten Abstufungen. Von den ca. $30 \mathrm{~cm}$ des Steppeninnern steigen die Zahlen in den Hochgebirgen und Küstenzonen zu Meterwerten, übersteigen sogar am Schwarzmeer 2,5 m (Rize), was auf monsunale Stau- und Steigregen zurückzuführen ist. Der kleinste Wert innerhalb 20 Jahren wurde, Irrtum vorbehalten, mit $14,8 \mathrm{~cm}$ in Konya gemessen (Max. $50 \mathrm{~cm}$, Mittel 32,2 cm).

Lebenswichtige Folgen für die türkische Landwirtschaft, der die Möglichkeit künstlicher Bewässerung fehlt, stehen hinter den Jahreskurven Fig. 4, Gefahr und Not hinter jenen der Minima.

Indessen sind nicht ausschlaggebend die minimalen Jahressummen allein, sondern vor allem die Frühjahrsniederschläge in der kritischen Wachstumsperiode, wozu wir als eindrückliches Beispiel Zahlen Huegrs für ein derart bedingtes Mißerntejahr der Konyagegend anführen:

$\begin{array}{lllll} & 1949 & \text { Jahresniederschlag } 351 \mathrm{~mm} & \text { Mai-Juni } 7 \mathrm{~mm} & \text { Weizenernte: } 141000 \mathrm{t} \\ \text { (Vergleich) } 1948 & \text { Jahresniederschlag } 357 \mathrm{~mm} & \text { Mai-Juni } 100 \mathrm{~mm} & \text { Weizenernte: } 717000 \mathrm{t}\end{array}$

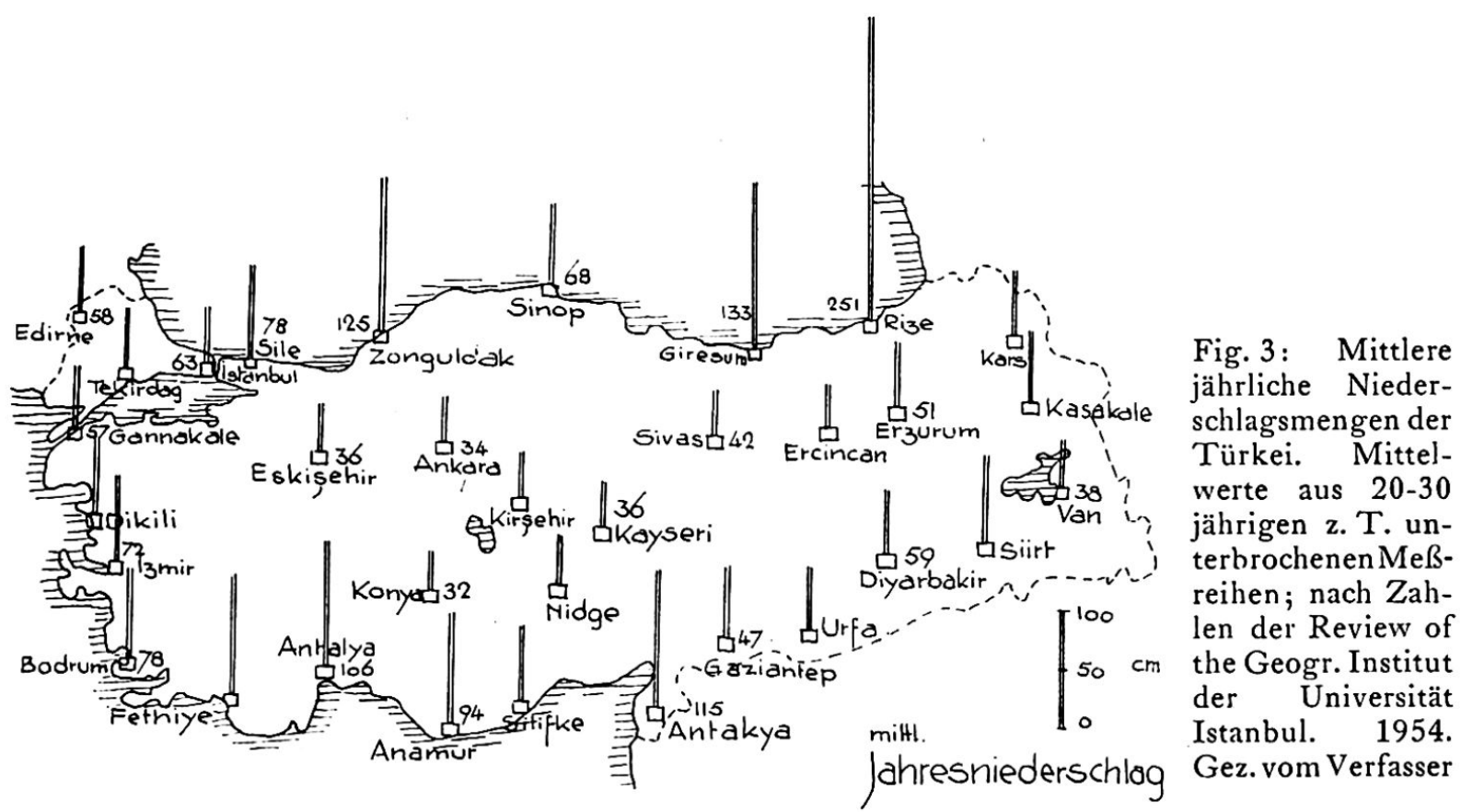

7 Ketin I. u. RoesLi F.: Makroseismische Untersuchungen über das nordwestanatolische Beben v. 18. März 1953. Ecl. Geol. Helv., Vol. 46, 2, 1953.

8 Tümertekin E.: div. klimatolog. Arbeiten über die Türkei, in Rundschau des Geograph. Institutes der Universität Istanbul, 1954-56. 

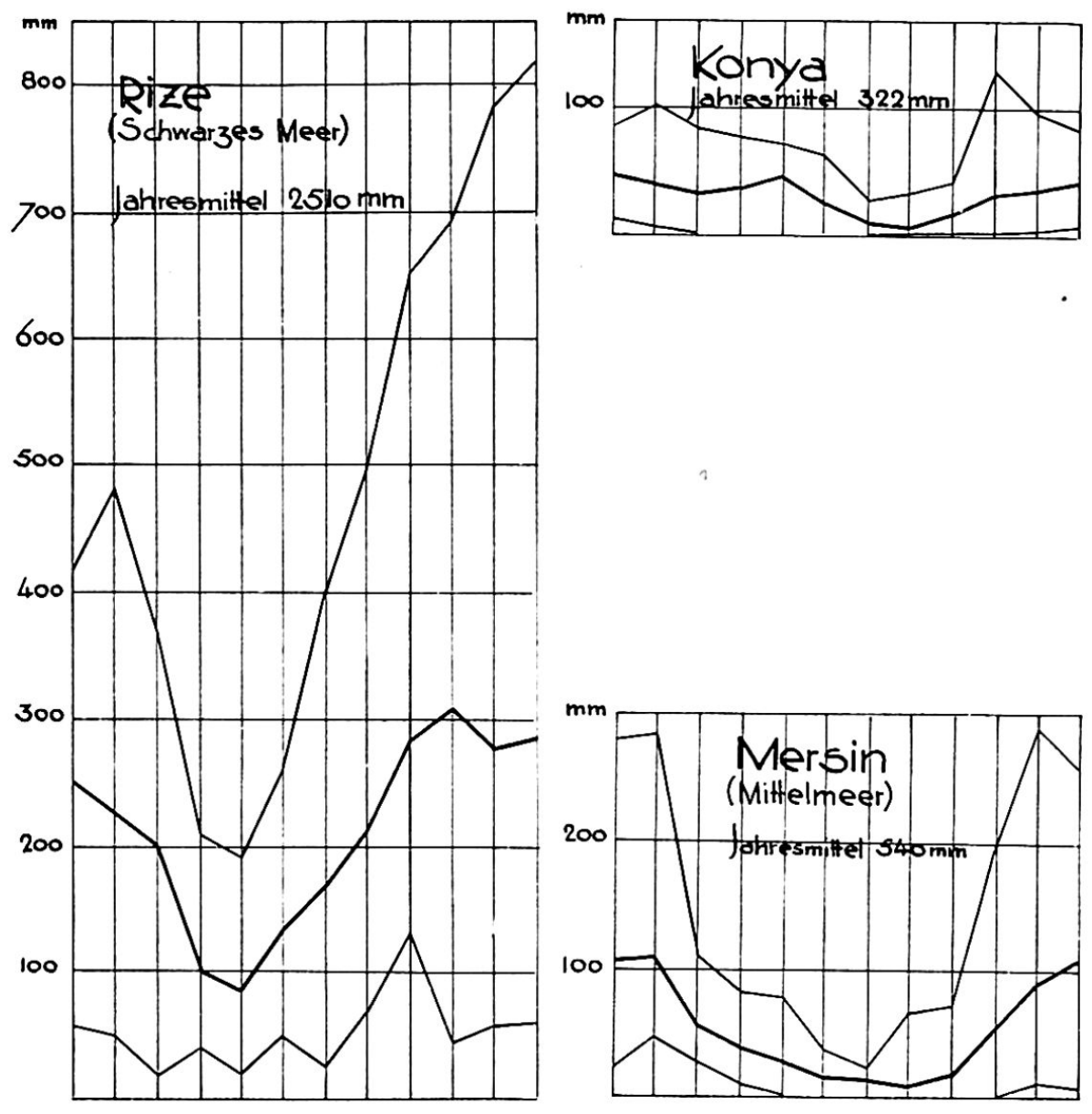

Fig. 4: Mittel, Maxima und Minima der Monatsniederschläge verschiedener türkischer Orte. Auffallend sind wie in Fig. 3 die großen regionalen Kontraste, bes. die Katastrophen repräsentierende Lage der der Minimumkurven.

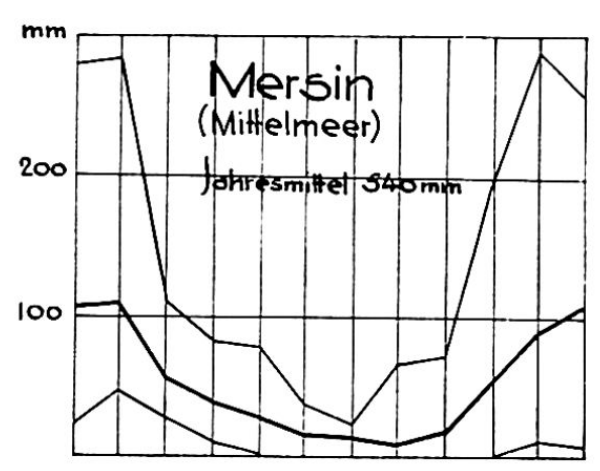

Die Steppenhochländer als die anatolische Charakterlandschaft haben neben geologischen (Tafelflächen) besonders klimatische Ursachen. Die regenreichen monsunalen Meerwinde stauen sich an den randlichen Gebirgen und strömen trocken und erhitzt ins Innere Kleinasiens. Das dortige aride Klima bedingt in bekannter Weise sowohl ganz bestimmte Böden und Gewässer, wie entsprechende, anpassungsfähige Pflanzen und Tiere.

Gebiete von über Schweizer Landesfläche bilden im Innern Anatoliens abflußlose Becken, dadurch, verbunden mit der großen Verdunstung, reichern sich in Böden und Seen Salze an. Die salzreichen Wasseransammlungen in den Becken trocknen in der kontinental klimatischen Sommerhitze nicht selten teilweise oder völlig aus (temporäre Seen). Die Salzwüste wird zudem bewirkt durch die in den ariden Bodentypen sich vollziehende Aufwärtszirkulation des mineralreichen Bodenwassers. HuEGI gibt für einen Grundwasserbrunnen im abflußlosen Konya ovasi die außergewöhnlich hohe Gesamthärte von $244^{\circ} \mathrm{fH}$ an (hoher Mineralgehalt bei uns $40-50^{\circ}$ franz. Härte). Der Salzgehalt des Tuz Gölü (türk., Salz-See), Typ eines echt asiatischen Salzsteppensees, beträgt 32,2\%. Er hat mehr als die doppelte Größe des Genfersees, wobei indessen bei derartigen Gegenüberstellungen Vorsicht geboten ist: mit der Anmut unserer Berg seen ist der öde, wenn auch eindrückliche Charakter des Salzsees nicht wohl zu vergleichen, der zumeist im Sommer ausgetrocknet ist und mithin eine bis $2 \mathrm{~m}$ dicke Salzkruste aufweisen soll, die sogar Pferdegespanne zu tragen vermöge.

Die folgenden Kapitel mögen einige Details zu verschiedenen hydrologisch-morphologischen Typen Anatoliens bringen. 
Aus den Taurusketten, 86 Straßenkilometer nw Konya, $1025 \mathrm{~m}$ hoch gelegen, reicht dieser See hinaus in die Steppenlandschaft. Nach LAHN ${ }^{9}$, Chaput und Blatt VI (Konya) ${ }^{10}$ der Türkiye Jeolojik Hartasi liegt der Çavusçu oder Ilgin Gölü in einem Grabenbruch. Die genannten Autoren wiesen eine allgemeine bruchtektonische Bedingtheit der zahlreichen Seen des westlichen Taurus (Seeufer und -axen), ähnlich der der Flußläufe, nach.

Auf den Karst als weitere Entstehungsursache weist die unterirdische Entwässerung des Çavusçu Gölü, wie anderer benachbarter Taurusseen, hin. Die verursachenden Kalke sind hier sehr unterschiedlichen Alters: Neogen und Permo-Karbon. Der Bruchgraben ist aufgefüllt mit «Alüviyonen» "zu einer flachen Seewanne. Der Zugang zum Wasser ist erschwert durch einen breiten, seichten Schilfverlandungsgürtel. Wassertemperatur am 22.10. $1958: 13,6^{\circ} \mathrm{C}$.

Typisch und schön ist der Austritt zweier Thermen auf der westlichen Störungslinie. Es war uns der Zeit und Unwegsamkeit wegen nicht möglich, die Ilica-Quelle $\left(28^{\circ}\right.$ C. $) 10 \mathrm{~km}$ nordwestlich Ilgin zu erreichen, erreichten schon mit Mühe nur im Zigeuner-Planwagen über einen Weg, der einem frischgepflügten Acker gleichkam, den See. In den Bädern der Hamam-Therme $2 \mathrm{~km}$ westlich Ilgin aber führten wir den Badeversuch am eigenen Leibe durch. Das Wasser hat nach unserer Messung die respektable Temperatur von $41,4^{\circ} \mathrm{C}$. Es soll Eisen und Schwefel enthalten und von allem Heilwirkung haben gegen rheumatische Krankheiten. Der Ertrag konnte nicht genau festgestellt werden, dürfte jedoch recht ansehnlich, in der Größenordnung einiger 100 Minutenliter sein. Das Wasser tritt am Fuße eines Kalkhügelzuges aus, es wird neben der tektonischen auch karsthydrologische Ursache vorhanden sein.

\section{KARSTQUELLEN IN DEN BERGGEGENDEN}

Quellen und laufende Brunnen sind (im Gegensatz zum Mangel an Oberflächenwasser in den Steppengebieten) für die gebirgigen Teile der Türkei typisch. Besonders laufende Brunnen (türk. Bezeichnung für Röhrenbrunnen: çesme) erkannten wir als gute Anzeiger von Landschaften größerer Reliefenergie oder deren Nähe. Bei den nachstehend besprochenen Quellen handelt es sich durchwegs um karstische Phänomene wo als Primärursachen Brüche und Klüftung mitwirkten.

Die Hamam-Therme auf einer Bruchlinie des nördlichen Taurusrandes, wenigstens teilweise wohl eine Karsterscheinung, und das zahlreiche Auftreten von Karstentwässerungen als für das Seengebiet des westlichen Taurus charakteristisches Phänomen, kamen bereits zur Sprache. In der nähern Umgebung von Antiochia, in den nördlichsten Libanon-Ausläufern, erweisen sich sodann zwei Quellen von besonderer Bedeutsamkeit als Karstquellen. Die berühmte Kirche St.Petrus über Antiochia, eine der ältesten christlichen Stätten, ist in eine Karstgrotte eingebaut. Unverkennbar ist bei diesem Einbau am Berghang die Flucht- und Schutzabsicht. In einer Ecke hinter dem mächtigen Marmoraltar tritt aus dem Kalk ein kleines Wasser aus. Früher dürfte ein größerer Abfluß die Höhle geschaffen haben, die für den Kirchenraum noch um einiges erweitert wurde.

Die Daphne-Quelle, $10 \mathrm{~km}$ südlich Antiochia, kündet sich an durch eine prächtige oasenhafte Pflanzenumgebung: Spanisch Rohr, Orangen, Palmen, Ahorn, Zedern. Die Römer richteten hier Bäder ein, woraus ein Teil jener hervorragenden Mo-

- LAHN E.: Türkiye Göllerinin Jeolojisi ve Jeomorfolojisi Hakkinda bir Etüt. (Contribution à l'Etude Géologique et Géomorphologique des lacs de la Turquie) MTAE Seri B, No. 12 (Publ. d. l'Inst. d'Etudes et d. Rech. Min. en Turquie) Ankara 1948.

10 Geologische Karte der Türkei 1:800 000, in 8 Blättern, Ankara $1942 \mathrm{f}$. 


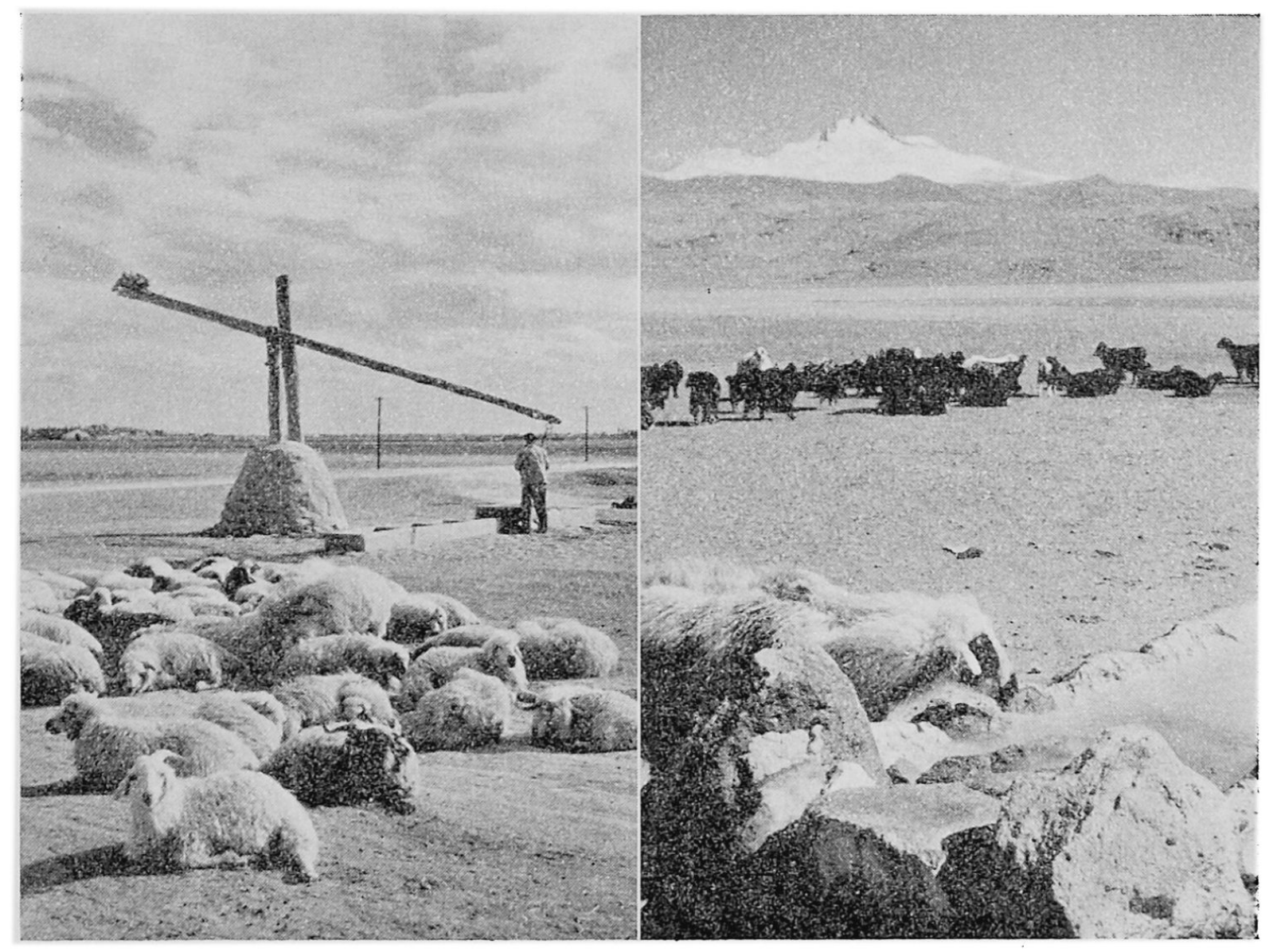

Links Fig. 5: Cigogner (Grundwasser-Schachtbrunnen, türk. kuyu) zwischen Ilgin und Konya, der die bisherige noch gebräuchliche Art der Wasserbeschaffung in den abflußlosen Steppengebieten zeigt. Rechts Fig. 6: Tränke in der Salzsteppe Zentralanatoliens (Incesu bei Kayseri) Schachtbrunnen, der bloß mit Seil und Kessel bedient wird. Im Hintergrund der Erciyas dag, $3916 \mathrm{~m}$.

Aufn. Val. Binggeli

saiken stammt, die das Museum von Antiochia zur größten derartigen Sammlung der Welt machen. Auch heute noch wird hier Trinkwasser gefaßt. Das Wasser erscheint aus stark ost-west geklüfteten und oberflächlich verkarsteten Kalken und Sintertuffen. An verschiedenen Stellen im und unterhalb des Quellgebietes, im ganzen zählten wir ca. $10 \mathrm{~W}$ asseraustritte, stehen gut aufgeschlossen bunte Mergel an, die sehr wahrscheinlich als undurchläßige Sammelbasis fungieren. Die einzelnen Teilquellen wiesen Erträge von einigen 100 Minutenlitern auf, (4 Temperaturen: $16,5^{\circ}$ C. $-16,6^{\circ} \mathrm{C}$ ). Die zahlreichen Rinnsale mäandern durch einen eindrucksvollen Hain alter mächtiger Eichen und Platanen und durch das romantische Grüngebäude von übermoosten Sinterbildungen.

\section{SALZWÜSTE UND SALZSEE S INCESU}

Wie der westlich des Erciyas Dag sich erstreckende Salzsumpf in seiner öden Monotonie und Ausgetrocknetheit etwas enttäuschend, obgleich eindrücklich, wirkte, so war umso besonderer und aufregender Art der Sandsturm, den wir in derselben Gegend erlebten. Beide aber charakterisieren landschaftlich-klimatisch-hydrologisch Hauptwesenszüge des Steppeninnern Anataoliens. Das Salzsumpfland (temporärer See) liegt $50 \mathrm{~km}$ nordwestlich Kayseri und war zur herbstlichen Zeit der Begehung oberirdisch fast völlig ausgetrocknet. Der durch hellen Lehmboden gekennzeichnete Raum (aus der Ferne täuscht er eine riesige Salzkruste vor) umfaßt eine Fläche von ca. $50 \mathrm{~km}^{2}$. Die Tiefe des temporären Sees scheint gering zu sein, wie die weite flache Wanne und 


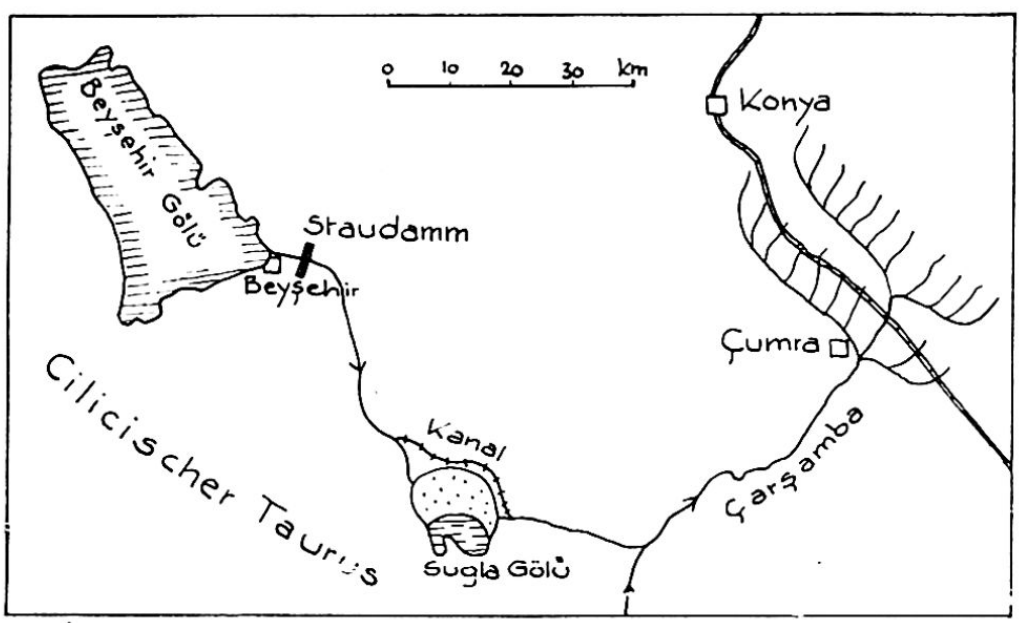

Fig. 7: Bewässerung im abflußlosen Konyabecken (Çumra) durch gestaute Wildwasser des Taurusgebirges.

frische Strandlinien am östlichen hügelgesäumten Ufer bezeugen; sie dürfte von der Größenordnung weniger Meter sein.

Im südlichen Teil des lehmigfeuchten Seebodens fanden sich sodann Salzkrusten, z. T. als nicht bestimmbare feinstengelige, weiße Auskristallisationen auf den Lehmpolygonen des ausgetrockneten und zerrissenen Grundes, z. T. wirklich als NaCl-Krusten. (Die Salze dürften eher als aus dem Erciyas-Massiv aus dem Taurus (Bulgar dag-Gegend) stammmen.) Der Lehmboden war durchzogen von prielartigen kleinen Kanälen mit spärlicher Wasserführung $\left(28^{\circ} \mathrm{C} . !\right)$. Wir beobachteten verschiedene Typen von Brunnen, alle auf einfachem Schacht-System (Türkische Bezeichnung für Schachtbrunnen: kuyu) basierend. Zahlreiche Schafherden mit Hunderten von schönen Tieren zogen tagsüber durch die Ebene südwärts und wurden getränkt (Fig. 6). In den Schächten bestimmten wir den Grundwasserspiegel als 2-4 m unter Terrainoberkante stehend, mit Zunahme der Tiefe proportional der Entfernung vom See.

Wie die stundenweite Wanderung über Lehm-, Salz- und Flechtenboden des Sumpfes, so war ein ungewöhnlich eindrückliches Erlebnis die Mittagszeit im Sandsturm. Die randlichen Ebenen und Plateaux am Salzsee hatten ganz das Gesicht einer Wüstensteppe oder Halbwüste: der Sandboden durchsetzt von spärlichen, mageren, harten Grasbüscheln, über Strecken sogar der Sand gehäuft zu kleinen Dünen mit schönen Rippelmarken. Gegen die heißtrockene Mittagszeit kam ein scharfer Südwind, wohl als Konvektionsströmung, d. h. als Bergwind vom Taurus her, auf. Fast 4 Stunden befanden wir uns nun im oft orkanartigen und Sandwolken dahertragenden Sturmwind. Bald waren wir bis auf die Haut mit Staubsand durchdrungen, der größere Sand schmerzte wie ein Sandgebläse auf Gesicht und Händen. Glücklicherweise ging unser Weg mit der Windrichtung, gegen den Sturm wäre das Vorwärtskommen, vor allem eine entsprechende Orientierung, unmöglich gewesen.

\section{DIE ABFLUSSLOSE BECKENLANDSCHAFT VON KONYA}

Wir erwähnten sie bereits als für die Gebiete Inneranatoliens charakteristische Landschaft. Das Problem der Wasserbeschaffung zu Trinkwasser- und Irrigationszwecken, um Steppenboden zu urbarisieren, tritt gerade in diesen Gegenden besonders deutlich hervor. Wohl sind schon Bohrungen zur Suche nach größern und guten Grundwasserschichten (im Vergleich zu denen der kuyus) abgeteuft worden. Für maschinelle Pumpwerke stellt sich eine neue Schwierigkeit, die der Energiebeschaffung. «Für die bessere Grundwasser-Nutzung bemühen sich aktiv verschiedene türkische Institutionen, so auch das Hydrologische Institut der Maden Fakultesi der technischen Universität in Istanbul» (HUEGI).

Der Cigogner (Fig. 5), der Grundwasserbrunnen alter, einfachster Manier mit langen Hebelarmen, Gegengewichten, Kette und Kessel ist auch heute noch das Zeichen 
dieser Gegenden. Auf 86 Kilometer zwischen Konya und Ilgin zählten wir an der Straße 74 Cigogners, neben bloß 8 laufenden Brunnen, die bereits die Randzone des Turus charakterisieren. Huegr gibt für das Wasserniveau unter Terrainoberkante Werte in der Größenordnung von Dekametern an. Wir erhielten zwischen Ilgin und Konya als Mittel aus 26 Beobachtungen $\mathrm{T}=5 \mathrm{~m}$ ( $2 \mathrm{~m}$ bis $15 \mathrm{~m})$. Die Temperaturen (ebenfalls Ilgin-Konya) zeigten sich als sehr konstant, von $14,3^{\circ} \mathrm{C}$ auf $14,7^{\circ} \mathrm{C}$ gegen Konya leicht ansteigend. Die $W$ asserqualität der kuyus läßt zumeist zu wünschen übrig, sowohl in chemischer wie bakteriologischer Hinsicht. Wasseranalysen mit Trokkenrückständen von weniger als $500 \mathrm{mg} / \mathrm{l}$ (die am ehesten mit Analysenzahlen schweizerischer Wässer verglichen werden können) machten nach den Messungen der erwähnten hydrologischen UNESCO-Mission bloß $15 \%$ aus. In Karaömerler $(30 \mathrm{~km}$ nordöstlich Konya) ergab ein Schachtwasser den extremen Wert von 8715,0 mg/1.

Eine große Bewässerungsanlage, bei Çumra südlich Konya ${ }^{11}$, die an Stelle von Grundwasser gestautes Wildwasser des Taurusgebirges benutzt, wurde zu Beginn des Jahrhunderts im Zusammenhang mit dem Bagdadbahn-Bau erstellt und in neuerer Zeit weiter ausgebaut. Diese Art der Wasserbeschaffung wird auch für andere Gebiete des Taurusrandes geprüft. Sie bringt als zusätzlichen Vorteil Abhilfe gegen die monatelangen Überschwemmungen der Beckenlandschaften durch die Tauruswildbäche. Deren sehr starke Unterschiedlichkeit der Wasserführung mag am Beispiel des Meram-Flusses bei Konya aufgezeigt sein, dessen Abflußzahlen zwischen $1 \mathrm{~m}^{3} / \mathrm{sek}$. und $60 \mathrm{~m}^{3} / \mathrm{sek}$. schwanken.

Fig. 7 zeigt das Bewässerungssystem von Çumra. Im Beysehir-See $\left(651 \mathrm{~km}^{2}\right)$ wird das zur Bewässerung der $120 \mathrm{~m}$ tiefer liegenden Konyaebene (Lykaonische Salzwüste) benötigte Wasser gestaut. Es gelangt durch einen Kanal, der den Sugla-See $\left(125 \mathrm{~km}^{2}\right)$ umfährt (dessen Großteil jahrweise temporär ausgetrocknet war und deshalb melioriert wurde) und durch das Bett des Çarsamba-Flusses auf die über mehrere $100 \mathrm{~km}^{2}$ ausgedehnten Steppenebenen. Im allgemeinen wird vom April-September jeweils innerhalb von 14 Tagen 2 Tage bewässert: die Çumra-Ebene (in frühern Betriebsjahren) mit $18,4 \mathrm{~m}^{3} / \mathrm{sek}$, der Seeboden des Sugla-Sees mit $3 \mathrm{~m}^{3} / \mathrm{sek}$. Daß auch hier nicht jede Schwierigkeit behoben, das «aride Wasserproblem» gelöst ist, erweist das typische Beispiel der Versalzungsschäden an den Kulturen nach dem ersten Weltkrieg, entstanden durch zu reichliche Bewässerung d. h. deren Folge, durch Spiegelanstieg des salzreichen Grundwassers. Dennoch aber ist Çumra ein durchgeführtes Beispiel dessen, was für weite Steppengebiete Anatoliens noch gesucht wird: Wasser - sei es Quell-, Grund-, See- oder Flußwasser - nicht bloß zu beschaffen, sondern zeitlich so zu beschaffen, daß v. a. in der ausschlagggebenden Vegetationsperiode das Lebenșelement in genügender Menge verfügbar ist.

\section{IMPRESSIONS SUR L'HYDROLOGIE ET LA MORPHOLOGIE DE L'ANATOLIE}

Le problème de l'eau est un des problèmes fondamentaux de l'Orient. Dans les vastes steppes de l'Anatolie centrale, la pénurie d'eau potable et d'irrigation, laquelle est necessaire en grande quantité, est très sensible. Mais il faut aussi considérer la signification symbolique et religieuse de l'eau: comme nous avons pu le constater, le musulman croyant suit strictement l'ancienne coutume des ablutions avant les cinq prières rituelles de la journée, imposée par le Coran. Notre voyage nous a montré les grands contrastes entre les divers paysages de l'Asie mineure, des côtes à la haute montagne, en passant par les steppes et les déserts de l'intérieur. Les moyennes des précipitations annuelles varient entre $251 \mathrm{~cm}$, mesurés sur la côte de la Mer Noire, et environ $30 \mathrm{~cm}$ dans les steppes. Les chaînes montagneuses qui encadrent l'Anatolie reçoivent d'importantes chutes de pluie; des fontaines (turc, çesme) et des sources karstiques sont typiques de ces régions, les puits (kuyu) des steppes. Ces paysages arides qui sont souvent des bassins sans écoulement, comme celui de Konya, sont caractérisés par des déserts et des lacs temporaires tous deux salés. - Près de Çumra au sud de Konya, existe un grand réseau d'irrigation qui utilise l'eau d'un barrage alimenté par les torrents du Taurus. Nombreux sont les projets semblables et d'autres utilisant les eaux souterraines.

11 Gerhardt P.: Bewässerung der Konyaebene. Zschr. f. Bauwesen. Berlin 1912. 